\title{
Monozygotic Twins Discordant for Immunoglobulin A Nephropathy Display Differences in DNA Methylation and Gene Expression
}

\author{
Min Wei ${ }^{a, b, c, d, e}$ Sijun Meng ${ }^{a, b, c, d, e}$ Sufang Shi ${ }^{a, b, c, d, e}$ Lijun Liu $^{a, b, c, d, e}$

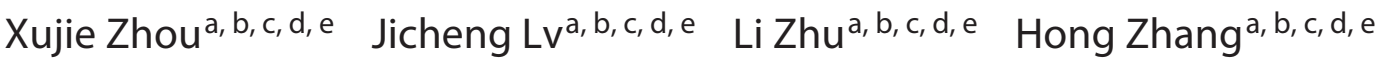

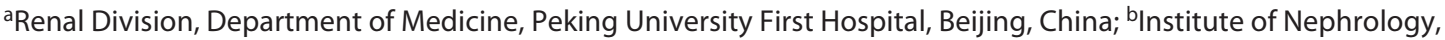 \\ Peking University, Beijing, China; ' Key Laboratory of Renal Disease, Ministry of Health of China, Beijing, China; ${ }^{\mathrm{d}}$ State \\ Key Laboratory of Chronic Kidney Disease Prevention and Treatment (Peking University), Ministry of Education,

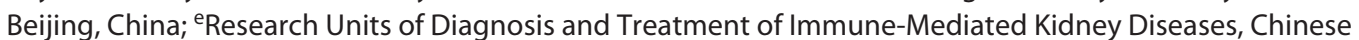 \\ Academy of Medical Sciences, Beijing, China
}

\section{Keywords}

Immunoglobulin A nephropathy · Monozygotic twins · DNA methylation · Gene expression

\begin{abstract}
Introduction: Immunoglobulin A nephropathy (IgAN) is the most common primary glomerulonephritis. It involves both genetic and environmental factors, among which DNA methylation, the most studied epigenetic modification, was shown to play a role. Here, we assessed genome-wide DNA methylation and gene expression profiles in 2 pairs of IgANdiscordant monozygotic (MZ) twins, in order to characterize methylation changes and their potential influences on gene expression in IgAN. Methods: Genome-wide DNA methylation and gene expression profiles were evaluated in peripheral blood mononuclear cells obtained from 2 IgAN-discordant $M Z$ twins. Differentially methylated regions (DMRs) and differentially expressed genes (DEGs) were detected, and an integrated analysis was performed. Finally, functional enrichment analysis was done for DMR-associated genes and DEGs. Results: Totally 521 DMRs were detected for 2 IgANdiscordant MZ twins. Among them, 9 DMRs were found to be mapped to genes that differentially expressed in $2 \mathrm{MZ}$
\end{abstract}

C 2020 The Author(s)

Published by S. Karger AG, Basel

This is an Open Access article licensed under the Creative Commons Attribution-NonCommercial-4.0 International License (CC BY-NC) (http://www.karger.com/Services/OpenAccessLicense), applicable to the online version of the article only. Usage and distribution for commercial purposes requires written permission. twins, indicating the potential regulatory mechanisms of expression for these 9 genes (MNDA, DYSF, IL 1R2, TLR6, TREML2, TREM1, IL32, S1PR5, and ADGRE3) in IgAN. Biological process analysis of them showed that they were mostly involved in the immune system process. Functional enrichment analysis of DEGs and DMR-associated genes both identified multiple pathways relevant to inflammatory and immune responses. And DMR-associated genes were significantly enriched in terms related to T-cell function. Conclusions: Our findings indicate that changes in DNA methylation patterns were involved in the pathogenesis of IgAN. Nine target genes detected in our study may provide new ideas for the exploration of molecular mechanisms of IgAN.

(C) 2020 The Author(s)

Published by S. Karger AG, Basel

\section{Introduction}

Immunoglobulin A nephropathy (IgAN) is the most common primary glomerulonephritis in the globe, characterized by the deposition of IgA1-containing immune complexes in glomerular mesangium [1]. It is an autoimmune inflammatory disease, in which aberrantly produced IgA1 play a key role [1]. IgAN is widely regarded 
as a common complex disease and is associated with the effects of many genetic and environmental factors. From IgAN with familiar aggregation, several IgAN-linked loci were reported. Moreover, a number of susceptibility loci of the disease have been identified from IgAN patients in recent genome-wide association studies. On the other hand, environmental factors such as dietary habits and personal hygiene were also shown to be relevant to IgAN development $[2,3]$. Given the complexity of IgAN phenotypes, to explore the intricate interaction between genetic and environmental factors is a very important approach to elucidate the precise pathogenesis of IgAN.

Epigenetic modifications, including histone modification, microRNAs, and DNA methylation, could influence phenotype through regulating gene expression without changing DNA sequence. It serves as an important bridge that associates environmental stimuli with genomic information. Previous studies showed that epigenetic modifications play important roles in multiple autoimmune diseases, such as type 1 diabetes [4] and rheumatoid arthritis (RA) [5]. As for IgAN, there is a lot of evidence, suggesting that DNA methylation participates in the development of IgAN [6-9]. For example, DNA hypermethylation in promoter region of Cosmc and the molecular chaperone of core $1 \beta 1,3$-galactosyltransferase in peripheral blood mononuclear cells (PBMCs) of IgAN patients were shown to be because of low expression of the gene and increased level of aberrantly glycosylated IgA1 [7].

Several previous studies have reported monozygotic (MZ) twins who were discordant for IgAN [10-12]. Since MZ twins develop from same fertilized eggs and share the same genome sequence, epigenetics is considered as a critical potential factor of this discordant disease status. And it helps in controlling potential confounding factors to use disease-discordant MZ twins in epigenetic studies. In this study, using 2 pairs of IgAN-discordant MZ twins, we detected genome-wide DNA methylation profile and gene expression profile to investigate the regulation of gene expression through DNA methylation changes (shown in Fig. 1) and to get a better understanding of the etiology of IgAN.

\section{Materials and Methods}

\section{Participants}

Two pairs of IgAN-discordant MZ twins were enrolled in the study from Peking University First Hospital. The first pair of twins were raised in different families since born, and the second pair of twins grew up together till they get married. To demon-

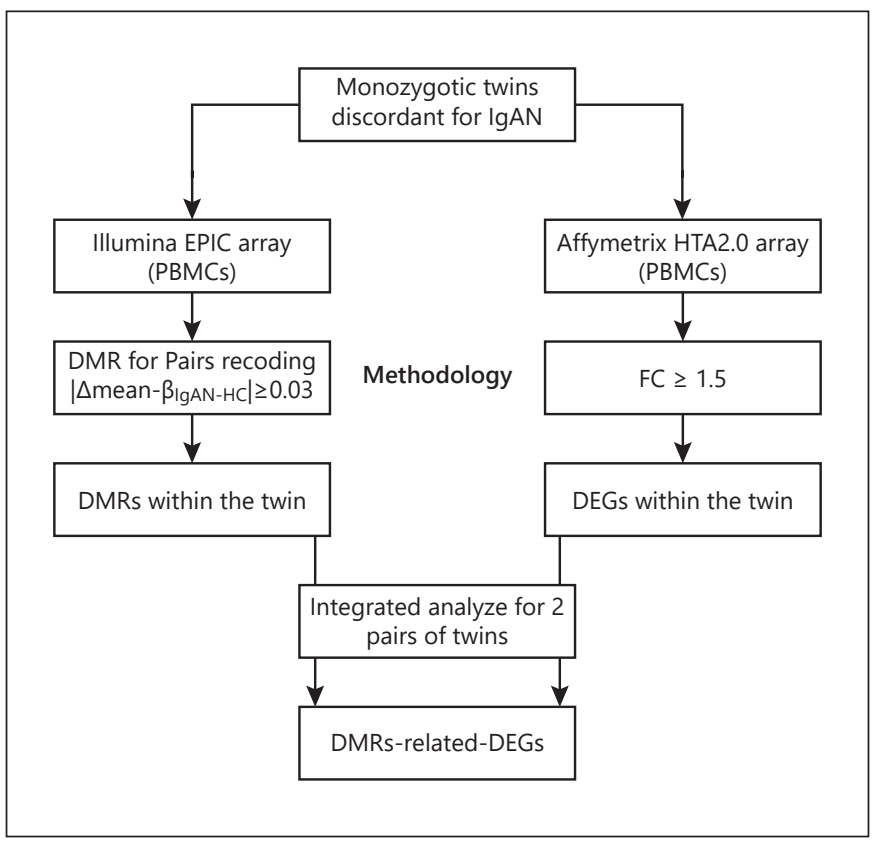

Fig. 1. Overview of the analysis process. IgAN, immunoglobulin A nephropathy; PBMCs, peripheral blood mononuclear cells; DMRs, differentially methylated regions; DEGs, differentially expressed genes; FC, fold change.

strate they were both identical twins, a zygosity test was performed using 20 short tandem repeat loci (D5S818, D13S317, D7S820, D16S539, VWA, TH01, TPOX, CSF1PO, D3S1358, D2S441, D2S1338, Penta E, D10S1248, D19S433, D21S11, D18S51, D6S1043, D8S1179, D12S391, and FGA) and the gender identification marker amelogenin. The diagnosis of IgAN was based on kidney biopsy, and secondary IgAN, IgA vasculitis, systemic lupus erythematosus, or other systemic autoimmune diseases were excluded. The kidney function of the healthy controls in twins was proved to be normal by blood and urine test at 2 different time points before blood collection. The main demographic and clinical characteristics of the IgAN patients in the twins are summarized in Table 1, and the kidney histology of both patients are shown in online suppl. Fig. 1 (see www.karger. com/doi/10.1159/000512169 for all online suppl. material). The research was carried out in accordance with the Declaration of Helsinki Principles. The written informed consent was obtained from all participants. The study was approved by the Peking University First Hospital Ethics Committees.

\section{DNA Extraction and DNA Methylation Profiling}

Whole blood was collected and PBMCs were isolated by means of density gradient centrifugation. Then, genomic DNA was extracted using a salting out method [13]. Bisulfite conversion of genomic DNA was performed using EZ DNA Methylation Kit (Zymo Research, Irvine, CA, USA) according to the manufacturer's instructions. Illumina Infinium MethylationEPIC BeadChips (EPIC array) (Illumina, San Diego, CA, USA) were subsequently used to establish the genome-wide DNA methyla- 
Table 1. Demographic and clinical features of IgAN patients in 2 monozygotic twins

\begin{tabular}{|c|c|c|}
\hline & $\begin{array}{l}\text { Pair 1-IgAN } \\
\text { patient }\end{array}$ & $\begin{array}{l}\text { Pair 2-IgAN } \\
\text { patient }\end{array}$ \\
\hline Sex & Male & Female \\
\hline Age at diagnosis & 53 & 48 \\
\hline Years discordant for IgAN at & & \\
\hline blood collection & 3 & 16 \\
\hline Systolic BP, mm Hg & 110 & 130 \\
\hline Diastolic BP, mm Hg & 70 & 90 \\
\hline $\mathrm{sCr}, \mu \mathrm{mol} / \mathrm{L}$ & 134 & 86.2 \\
\hline $\mathrm{eGFR}, \mathrm{mL} / \mathrm{min} / 1.73 \mathrm{~m}^{2}$ & 51.46 & 71.32 \\
\hline Proteinuria, 24 h; g/d & 0.79 & 1.45 \\
\hline Oxford classification & M1E0S1T1C2 & M1E0S1T1C0 \\
\hline
\end{tabular}

The above clinical data were collected at initial diagnosis. IgAN, immunoglobulin A nephropathy.

tion profiles, and the array was scanned using Illumina iScan. Samples of both twins were processed within the same batch to avoid batch effect.

\section{RNA Extraction and Gene Expression Profiling}

RNA was extracted using RNeasy Mini Kit (Qiagen, Stockach, Germany). Then, $100 \mathrm{ng}$ of RNA was processed and hybridized to Affymetrix Human Transcriptome Array 2.0 (HTA2.0). After washing and staining, the array was scanned using Affymetrix Scanner 3000. Samples of both twins were processed and assayed in the same batch.

\section{EPIC Array Data Preprocessing}

The raw intensity files (accessible as GSE151456 at GEO repository) were imported into $\mathrm{R}$ using minfi packages [14]. A $\beta$ value was calculated for each $\mathrm{CpG}$ site, which falls between 0 and 1 and indicates the level of methylation ( 0 refers to unmethylated status and 1 refers to fully methylated status). R package minfi [14], wateRmelon [15], and DMRcate [16] were used for quality control and data preprocessing. Samples with mean detection $p$ value $>0.01$ or bisulfite conversion rate $<95 \%$ were considered unqualified. Normalization of the data was conducted using the preprocessQuantile() function in minfi. The probes that met the following criteria were filtered out: (1) the probes with detection $p$ value $>0.01$ or low bead count ( $<3$ beads) in 1 or more samples, $(2)$ nonCpG probes, (3) cross-reactive probes, (4) probes that measure SNPs, and (5) probes on the sex chromosomes.

The composition of B cells, CD4 T cells, CD8 T cells, monocytes, granulocytes, and natural killer cells was estimated using estimateCellCounts() function from minfi package and a paired $t$ test was performed to check whether the cell composition was similar within each twin pair.

\section{Differential DNA Methylation Analysis}

After data preprocessing, a within-pair Pearson correlation was first performed for each twin pair to detect the difference in global DNA methylation status. To detect differentially methyl- ated regions (DMRs), R package DMRforPairs [17] was then used to assign each single $\mathrm{CpG}$ site into different regions based on the following criteria: (1) a region of interest must contain no less than $4 \mathrm{CpG}$ sites and (2) the distance between neighboring $\mathrm{CpG}$ sites in the same region should be within $200 \mathrm{bp}$. A mean $\beta$ value (mean- $\beta$ ) of all CpG sites within a region was calculated for each region subsequently. DMRs were identified for each twin pair based on an absolute mean methylation difference between the IgAN patient and the healthy control $>0.03$ ( $\mid \Delta$ mean- $\beta \mid$ $\geq 0.03)$. Probe annotation was performed according to hg19, and DMRs were annotated to the nearest genes using ChIPseeker [18] package. Gene promoter regions were defined as regions within $2 \mathrm{~kb}$ upstream and downstream of transcription start sites (TSS2000). In the current study, we focused on the following 2 types of DMRs: promoter-DMRs (regions mapped to promoter regions) and gene-body-DMRs (regions mapped to $3^{\prime}$ UTR or gene bodies). All the above analysis was performed using $\mathrm{R}$ (v3.6.2).

\section{Differential Expression Analysis and Combined Analysis with \\ DMRs}

Data preprocessing and the downstream statistical analysis of the gene expression array were all carried out using TAC (v4.0.2) software (accessible as GSE151454 at GEO repository). During quality control, the quality of starting sample, hybridization efficiency, and the target labeling process were estimated. Samples with a pos versus neg AUC value $<0.80$ were filtered out. The raw expression signals were log transformed and normalized with SSTRMA method. For genes that matched to $>1$ transcript clusters, the expression signals of all transcript clusters mapped to that gene were averaged. The differentially expressed genes (DEGs) were identified by fold change (FC) $\geq 1.5$ thresholds.

As DNA methylation status could influence the expression level of the associated genes, we screened out DEGs to which DMRs were annotated as our candidate genes (DMR-related DEGs). For promoter-DMRs, we only screened for genes of which expression levels and DNA methylation levels altered in the opposite directions. For gene-body-DMRs, we only screened for genes of which expression levels and DNA methylation levels altered in the same directions.

\section{Further Bioinformatics Analysis of DMR-Related DEGs}

To further assess the role of detected DMR-related DEGs in IgAN, protein-protein interaction network of DMR-related DEGs was constructed using STRING (v11.0) online database and Cytoscape software (v3.6.0). Interaction score of 0.4 was set as the threshold, and 10 more predicted interaction partners were included for network construction. The biological process analysis of DMR-related-DEGs was performed using BiNGO (v3.0.4) app in Cytoscape.

\section{Functional Enrichment Analysis}

Gene Ontology (GO) enrichment and Kyoto Encyclopedia of Genes and Genomes (KEGG) pathway analysis were performed for the DMR-annotated genes and DEGs separately using WebGestalt software [19]. ORA method was employed to identify statistically significant enrichments (adjusted $p$ value $<0.05$ ). The background in the analysis consists of all genes, which were annotated by the EPIC array or HTA2.0 array. 


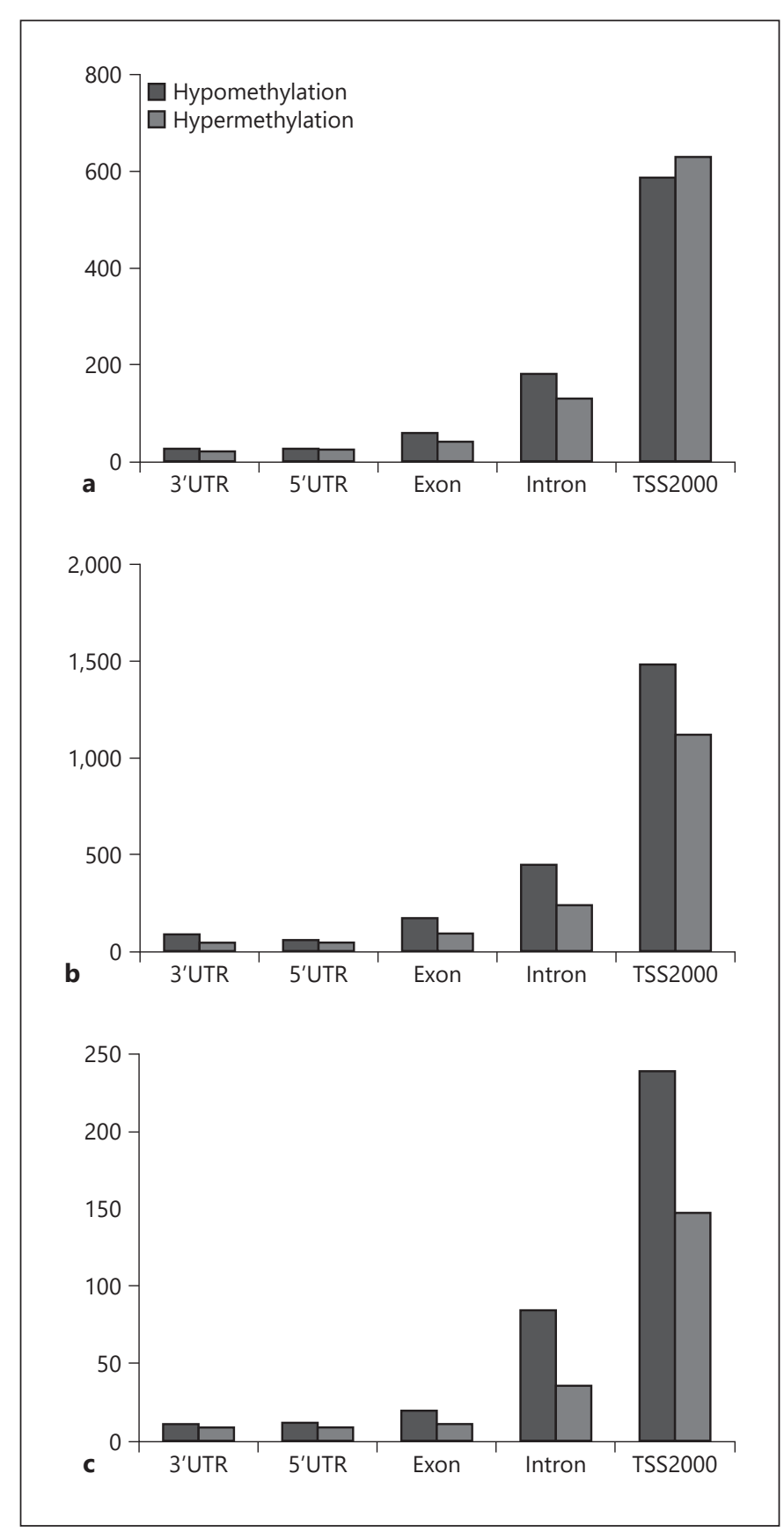

Fig. 2. The distribution of DMRs on the genome. Within-pair DMRs were divided into 2 types (hypermethylation and hypomethylation) and further classified into 5 categories based on their locations. a The distribution of 1,647 DMRs in pair 1 . b The distribution of 3,580 DMRs in pair 2. c The distribution of 551 DMRs shared by both twin pairs. DMRs, differentially methylated regions.

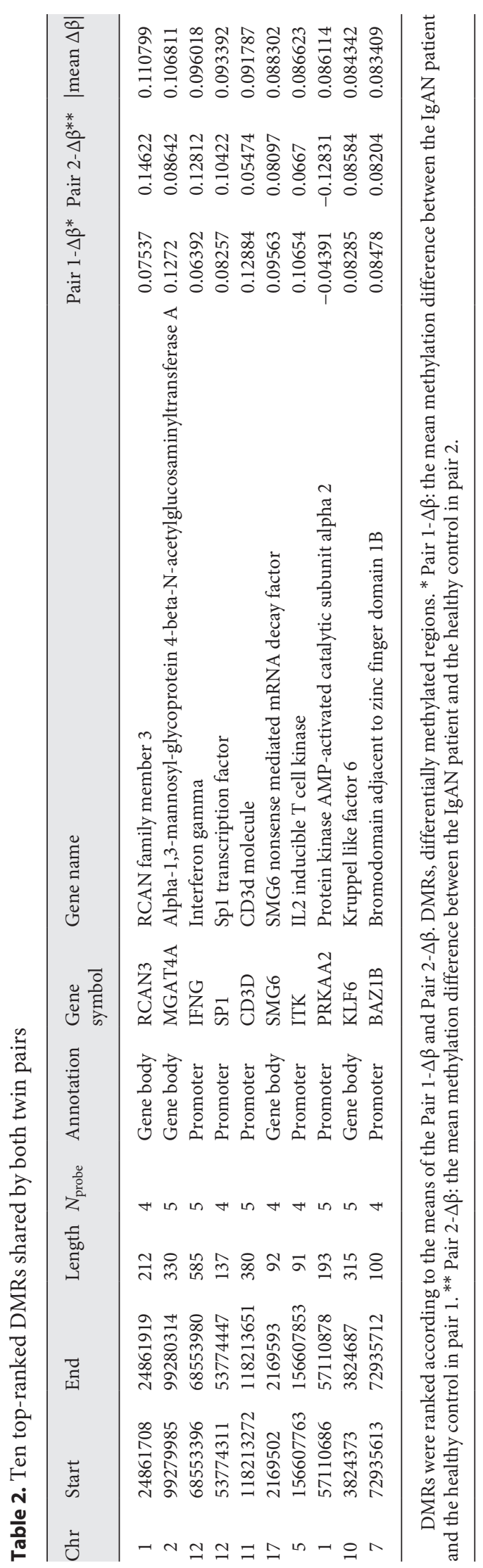


Table 3. Nine DMR-related DEGs shared by both twin pairs

\begin{tabular}{|c|c|c|c|c|c|c|c|c|}
\hline \multirow[t]{2}{*}{ Gene } & \multicolumn{4}{|c|}{ DMRs } & \multicolumn{2}{|l|}{ Pair 1} & \multicolumn{2}{|l|}{ Pair 2} \\
\hline & $\operatorname{chr}$ & start & end & annotation & FC & $\Delta \beta_{\text {IgAN-HC }}$ & FC & $\Delta \beta_{\text {IgAN-HC }}$ \\
\hline$M N D A$ & 1 & 158800873 & 158801271 & Promoter & $1.62 \uparrow$ & -0.03372 & $1.51 \uparrow$ & -0.03372 \\
\hline DYSF & 2 & 71680559 & 71680721 & Promoter & $2.14 \uparrow$ & -0.0438 & $2.55 \uparrow$ & -0.07977 \\
\hline$I L 1 R 2$ & 2 & 102608155 & 102608349 & Promoter & $3.13 \uparrow$ & -0.033 & $2.25 \uparrow$ & -0.05176 \\
\hline TLR6 & 4 & 38859587 & 38859770 & Promoter & $2.46 \uparrow$ & -0.05536 & $1.52 \uparrow$ & -0.03412 \\
\hline TREML2 & 6 & 41168801 & 41169049 & Promoter & $2.13 \uparrow$ & -0.03245 & $1.92 \uparrow$ & -0.03122 \\
\hline TREM1 & 6 & 41254818 & 41255090 & Promoter & $3.01 \uparrow$ & -0.0345 & $1.96 \uparrow$ & -0.03396 \\
\hline IL32 & 16 & 3115552 & 3115809 & Promoter & $-2.46 \downarrow$ & 0.06432 & $-1.95 \downarrow$ & 0.057172 \\
\hline S1PR5 & 19 & 10628535 & 10629035 & Promoter & $-1.55 \downarrow$ & 0.031617 & $-1.59 \downarrow$ & 0.03392 \\
\hline ADGRE3 & 19 & 14785593 & 14785849 & Promoter & $3.78 \uparrow$ & -0.03154 & $2.33 \uparrow$ & -0.03039 \\
\hline
\end{tabular}

DMRs, differentially methylated regions; DEGs, differentially expressed genes; FC, fold change.

\section{Results}

\section{Genome-Wide DNA Methylation Profiling}

Both twins studied in this research were proved to be MZ twins through zygosity test. None of the samples was excluded during quality control. As we used PBMCs as source of DNA for methylation profiling and variation of cell composition may have an influence on DNA methylation level. The blood cells composition was estimated and proved to be similar within each twin pair (online suppl. Table 1). We first tried to compare the DNA methylation status within $2 \mathrm{MZ}$ twins at a global level. As predicted, Pearson's correlation coefficients were both $>0.99$ within 2 twins (pair 1: $r=0.995$; pair 2: $r=0.991$ ), suggesting that the global DNA methylation patterns were almost the same within both twins and IgAN is potentially not associated with global changes in DNA methylation status.

\section{DNA Methylation Changes Shared by Both Twins}

To investigate DNA methylation changes associated with putative pathogenetic genes, we screened for common DMRs shared by 2 twins. In this study, we focused on the regions mapped to gene promoters and gene bodies. As we all know, IgAN is a complicated disease that involves many different genetic and environmental factors, we set a relatively low threshold for DMRs identification so as to detect subtle but potentially significant DNA methylation changes. Regions that contain no less than $4 \mathrm{CpG}$ sites with mean methylation difference $>0.03$ ( $\mid \Delta$ mean- $\beta$ IgAN-HC $\mid \geq 0.03$ ) were considered as DMRs. In total, 1,647 DMRs and 3,580 DMRs were detected for pair 1 and pair 2, respectively. The distribution of DMRs on the genome is shown in Figure 2. Most of the DMRs were located in the promoter regions (73.1\% for pair 1 and $70.9 \%$ for pair 2 ), and less were found in gene bodies (22.9\% for pair 1 and $24.2 \%$ for pair 2) in both twins. We next looked into the DMRs that shared by both twin pairs (online suppl. Table 2). Of 551 common DMRs, more than half (69.3\%) were located in the promoter regions, and more DMRs were hypomethylated than DMRs that were hypermethylated (shown in Fig. 2c). The 10 top-ranked common DMRs are listed in Table 2. The first one is regulator of calcineurin 3 (RCAN3), an inhibitor of calcineurin. It was shown to be involved in Th17 cell differentiation in RA [20].

\section{DEGs and Combined Analysis with DMRs}

As DNA methylation is an important epigenetic mechanism that regulates gene expression, genome-wide gene expression profiling was performed for $2 \mathrm{MZ}$ twins using microarrays to screen for DEGs that might be associated with changes in DNA methylation. 2,842 genes and 520 genes passed filtering with FC $>1.5$ threshold for pair 1 and pair 2, respectively. There were 251 common DEGs shared by both twins (online suppl. Table 3 ).

DNA methylation of gene promoters has been shown to be negatively associated with gene expression while gene body methylation was usually positively correlated to gene expression. Based on this rule, we next performed the integrated analysis of DEGs and DMRs and finally screened out 9 DMR-related DEGs shared by 2 twins (Table 3). Protein-protein interaction network was then constructed for them using STRING online database (shown in Fig. 3). The biological process anal- 
Fig. 3. PPI network of 9 DMR-related DEGs and 10 predicted interaction partners constructed using STRING. Black nodes represent 9 DMR-related DEGs and gray ones were predicted interaction partners of them. The boldness of lines represents the combined score of the interaction. PPI, protein-protein interaction; DMR, differentially methylated region; DEGs, differentially expressed genes.

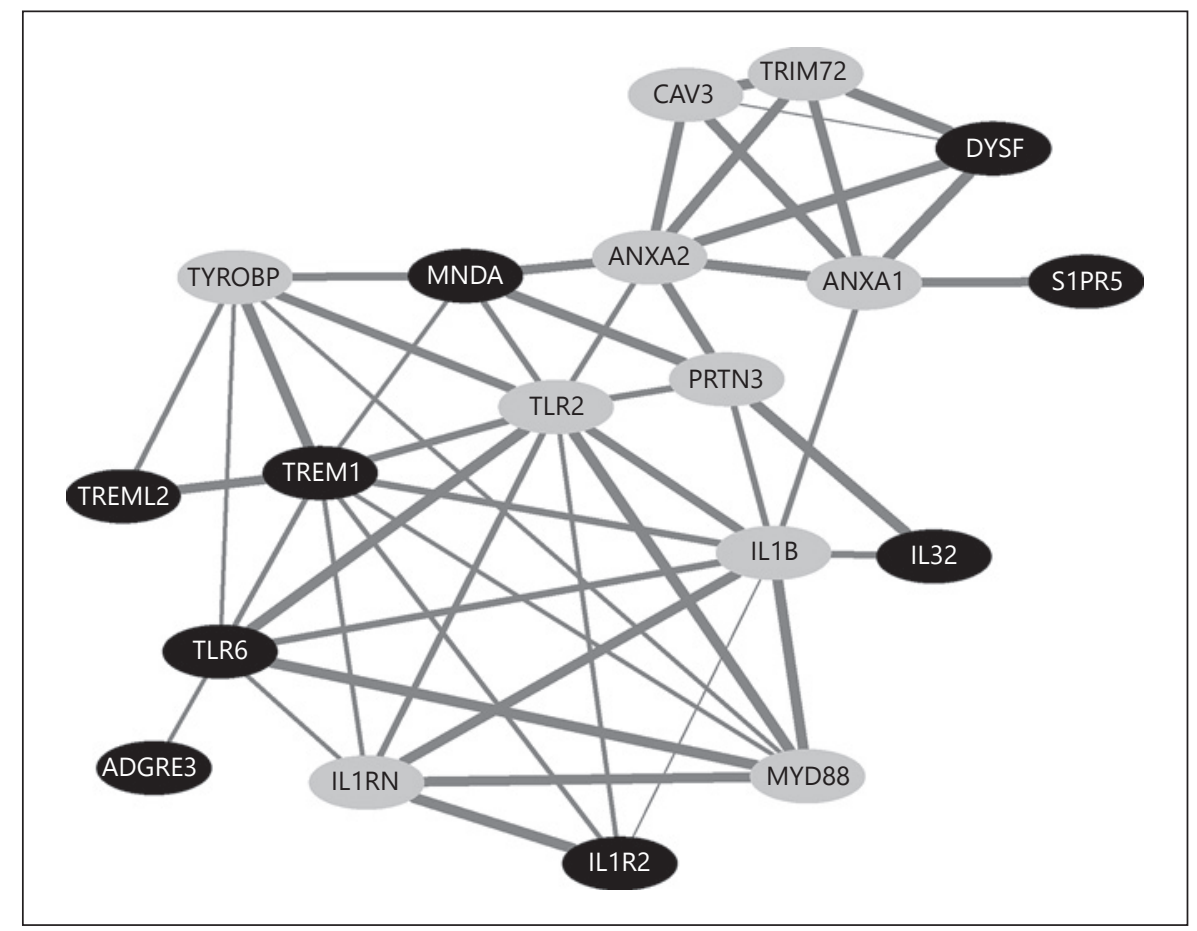

ysis of 9 DMR-related DEGs showed that the most significant BP was immune system process.

\section{Functional Enrichment Analysis}

To better understand the molecular mechanisms associated with the pathogenesis of IgAN in the twins, functional enrichment analysis was done for 521 DMR-associated genes and 251 common DEGs (shown in Fig. 4; online suppl. Tables $4-7$ ). Figure 4 shows the significantly enriched GO terms and KEGG pathways with $p$ value $<0.01$.

The result of GO enrichment analysis using DMR-associated genes indicated that most significantly enriched terms were leukocyte differentiation, T-cell activation, and regulation of leukocyte activation. According to KEGG pathway analysis, DMR-associated genes were mostly related to T-cell receptor signaling pathway, Th17 cell differentiation, and allograft rejection.

The GO enrichment analysis of 251 DEGs showed that DEGs were most significantly associated with neutrophilmediated immunity, granulocyte activation, and secretory granule membrane. The KEGG pathway enrichment analysis revealed that DEGs were largely enriched in ribosome biogenesis in eukaryotes, ribosome, and natural killer cell-mediated cytotoxicity.

Epigenetic and Transcriptomic Study in IgAN-Discordant Monozygotic Twins

\section{Discussion}

In the present study, we did a combined analysis of DNA methylation and gene expression profiling in 2 pairs of IgAN-discordant MZ twins. To the best of our knowledge, this is the first study to explore genome-wide DNA methylation changes and its association with gene expressions in IgAN. Using disease-discordant MZ twins in the study of epigenetics makes it easy to control potential confounding factors, such as age, gender, and genetic background. We finally screened out 9 DMR-related DEGs shared by both twins, which may provide new ideas for the exploration of molecular mechanisms of IgAN. The biological functional analysis of 9 DMR-relatedDEGs indicated that these genes were mainly involved in immune system process.

Interleukin 1 receptor type 2 (IL1R2) is a decoy receptor for IL-1 and was shown to be expressed on neutrophils, B cells, monocytes, and macrophages. Its main function is to negatively regulate IL-1 signaling pathway [21]. Recently, polymorphisms in IL1R2 gene were reported to be associated with the risk of IgAN in the Han Chinese population [22]. In the present study, we detected the hypomethylation of the promoter of IL1R2 gene together with its higher expression in IgAN patients compared to their healthy sib- 


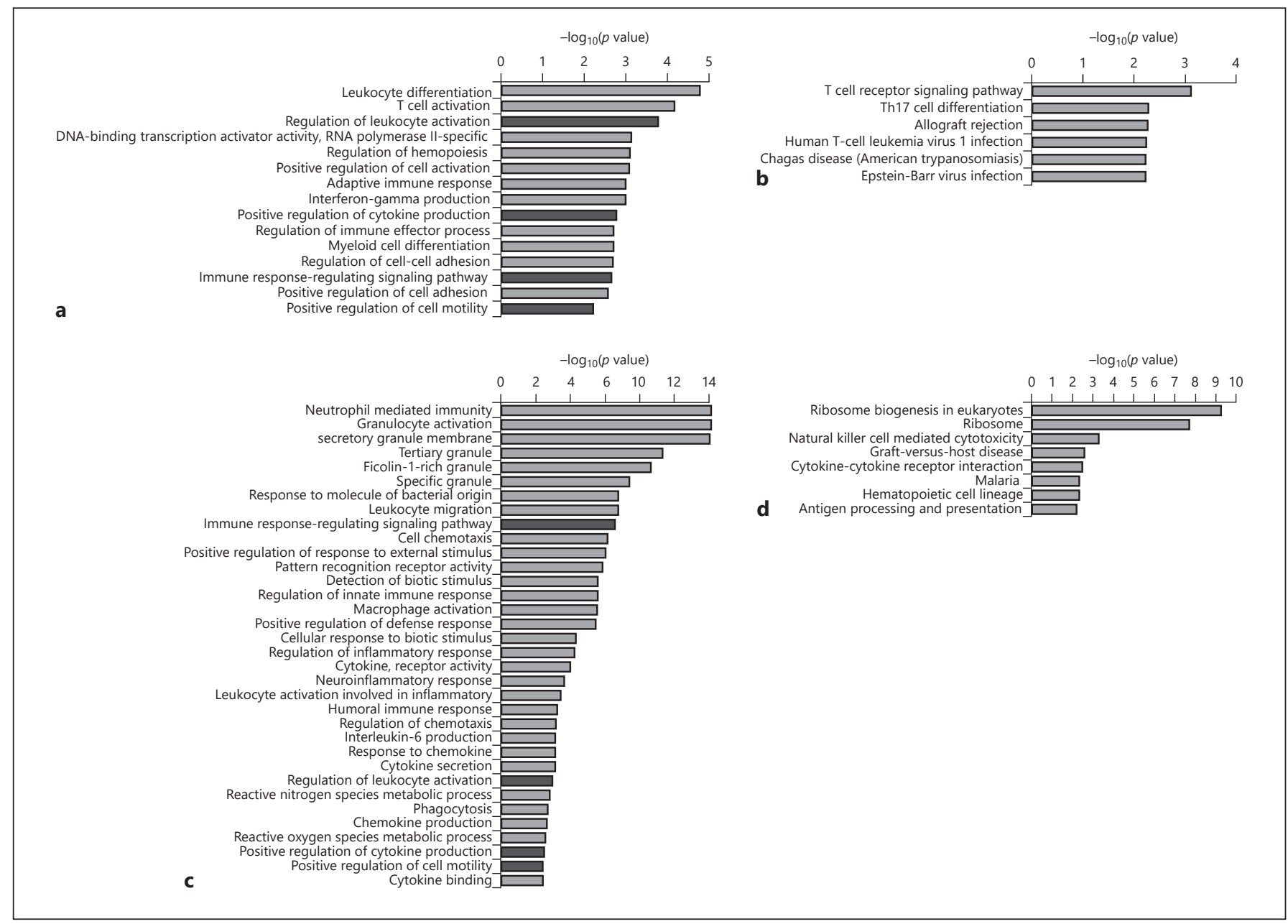

Fig. 4. GO and KEGG pathway enrichment analysis of $521 \mathrm{DMR}-$ associated genes and 251 DEGs. Enriched terms are ranked based on the enrichment $p$ values calculated by WebGestalt software and terms with adjusted $p$ value $<0.01$ are shown in bar charts above. The black bars represent the common pathways shared by DMRassociated genes and DEGs. a Enriched GO terms of DMR-associ- ated genes. b Enriched KEGG pathways of DMR-associated genes. c Enriched GO terms of DEGs. d Enriched KEGG pathways of DEGs. GO, Gene Ontology; KEGG, Kyoto Encyclopedia of Genes and Genomes; DMR, differentially methylated region; DEGs, differentially expressed genes. lings, indicating the potential role of IL1R2 in the development of IgAN.

TLR6 was another putative candidate gene found in this study and belongs to Toll-like receptor (TLR) family. TLRs are an important kind of pattern recognition receptors expressed in a diverse range of cells, including macrophages, dendritic cells, neutrophils, and B cells [23]. It was reported that TLR4 was upregulated in PBMCs of patients with IgAN [24]. Activation of TLR9/MyD88 signaling pathway was shown to be associated with severe renal injury in IgAN based on a murine model [25]. In addition, a recent study showed that intrarenal staining of TLR4, 7, 8, and 9 was more intense in IgAN patients than in healthy controls, and the intensity of TLR4 and TLR9 in kidney tissue was associated with disease severity [26]. All these data indicated the involvement of TLRs in IgAN. There was no evidence that TLR6 was involved in IgAN so far, but the association of TLR6 with other inflammatory disease was suggested in the previous studies, such as inflammatory bowel diseases [27]. Hence, it would be worthwhile to further investigate the role of TLR6 in IgAN.

Another gene of potential interest is triggering receptor expressed on myeloid cells-1 (TREM1). TREM1 is a pro- 
inflammatory receptor mainly expressed by neutrophils and monocytes. It has been shown to be highly expressed in some inflammatory diseases, including RA [28] and inflammatory bowel diseases [29]. The activation of TREM1 signaling pathway would promote the release of multiple cytokines and chemokines, resulted in amplification of inflammatory responses [30]. Our previous studies found that IgA1-containing immune complexes from IgAN patients could upregulate the expression of TREM1 in cultured mesangial cells and elevated level of its soluble form (sTREM1) in the urine of IgAN patients was correlated with severe clinical and pathological manifestations [31, 32]. Besides, a recent study showed that the increase of TREM1 mRNA expression level in peripheral leukocytes of patients with Alzheimer's disease was significantly correlated with the hypomethylation of the gene promoter [33]. Here in our twin study, we detected the hypomethylation of TREM1 gene promoter and the upregulation of gene expression in IgAN patients, indicating change of DNA methylation status of TREM1 may play a role in the disease. Moreover, TREML2, another member of TREM family, showed the same change in RNA expression and DNA methylation as TREM1 in our study. Regarding the left 5 DMR-related DEGs, currently there is no direct evidence indicating their association with IgAN. Therefore, further studies are needed to provide more information.

Functional enrichment analysis of DEGs and DMRassociated genes identified multiple pathways relevant to inflammatory and immune responses. These findings are in line with previous study that abnormalities of immune system play an important role in the pathogenesis of IgAN1 [1]. According to the results, DMR-associated genes were significantly enriched in GO terms and KEGG pathways related to T-cell function (GO term: 0042110, T-cell activation; KEGG: hsa04660, T-cell receptor signaling pathway; hsa04659, Th17 cell differentiation). The role of T cells in IgAN was recently deeply investigated, and it has been shown that several T-cell subtypes, such as Th2, Th17, and Th22, in the circulation of patients with IgAN were overactivated [34]. Specifically, Th17 polarization and Th17/Treg disequilibrium were shown to be involved in IgAN, and IL17 secreted by Th17 cells were suggested to promote the synthesis of aberrantly glycosylated IgA1 $[35,36]$. Furthermore, epigenetic changes were shown to regulate T-cell function in patients with IgAN. A recent study showed low expression of microRNA-155 in PBMCs of IgAN patients was associated with T lymphocyte subgroup drifting [37]. A genome-wide DNA methylation profiling using CD4+ $\mathrm{T}$ cells revealed that aberrant methylation of DUSP3, TRM27, and VTRN2-1

Epigenetic and Transcriptomic Study in IgAN-Discordant Monozygotic Twins genes was associated with reduced TCR signal strength of the CD4+ T cells in IgAN patients [6].

Despite the advantages of using disease-discordant MZ twins, there were several limitations of this study. First one is that our sample size was limited by the extremely low incidence of MZ twins discordant for IgAN. Second, the patients in the twins had been taking therapeutic drugs for a long time before blood collection, so it is hard to tell whether changes in DNA methylation come from drug treatment or not. Third, although we isolated PBMCs from circulation for DNA methylation profiling and compared the differences in $\mathrm{MZ}$ twins discordant for IgAN, the lack of other tissue samples (gut/kidney/bone marrow cells), especially from the healthy individuals from both twins, prevented our exploration in other cells, which also play important roles in IgAN pathogenesis. Fourth, it is little difficult to determine our identified DMR-related DEGs as the cause or the consequence of IgAN. Further studies are needed to evaluate the factors influenced the DMRs, as well as the causal effect of the DMR-related DEGs on IgAN pathogenesis, either by mQTL (methylation quantitative trait locus) analysis or by functional studies in cell or animal models. Fifth, the 2 pairs of twins had been discordant for IgAN for 3 and 16 years until the recruitment in our study. Since IgAN is a slowly progressed disease and big variations are often reported in the patients of IgAN pedigrees, it is still possible that the healthy individuals in the twins may develop kidney disease at a late stage. And the twins will be regularly followed up in the future. Sixth, for our identified 9 DMR-related DEGs, we did not perform validation experiments in other MZ twins discordant for IgA nephropathy as well as in these 2 pairs of twins by either bisulfate DNA sequencing or qPCR. This is largely limited by the recruitment of this special twins, which is extremely rare. Seventh, besides DNA methylation, other epigenetic regulation mechanisms, such as microRNAs, may also contributed in IgAN, which should be investigated in the future.

In summary, our study provides a great chance to understand epigenetic mechanisms involved in IgAN through integrated analysis of genome-wide DNA methylation and RNA expression profiles in IgAN-discordant MZ twins. Our results of enrichment analysis highlighted the role of immune and inflammatory response in the pathogenesis of IgAN. Nine DMR-related DEGs were identified as potential epigenetic biomarkers for IgAN, and they were shown to be mostly involved in immune system process. The underlying mechanisms about how these genes take part in IgAN still need further research in the future. 


\section{Acknowledgement}

We gratefully acknowledge the participants in our study for their cooperation.

\section{Statement of Ethics}

The research was carried out in accordance with the Declaration of Helsinki Principles. The written informed consent was obtained from all participants. The study was approved by the Peking University First Hospital Ethics Committees (2018KEYAN099).

\section{Conflict of Interest Statement}

The authors have no conflicts of interest to declare.

\section{Funding Sources}

This project was supported by grants from the National Science Foundation of China (81922013, 81970598), National Science Foundation of Beijing (7202206), National Key Research and Development Program of China (2019YFC2005000), Beijing Science and Technology Plan Project of China (D181100000118003, Z161100000516005), Chinese Academy of Medical Sciences Research Unit, Peking University (No. 2019RU023), and CAMS Innovation Fund for Medical Sciences (2019-I2M-5-046).

\section{Author Contributions}

M.W., S.M., and L.Z. were involved in the conception and design of the experiments. S.M. recruited patients and performed experiments. M.W. analyzed data and drafted the manuscript. S.S., L.L., X.Z., J.L., L.Z., and H.Z. critically revised the manuscript. All authors approved the final version of the manuscript.

\section{References}

1 Lai KN, Tang SC, Schena FP, Novak J, Tomino $\mathrm{Y}$, Fogo AB, et al. IgA nephropathy. Nat Rev Dis Primers. 2016 Feb 11;2:16001.

2 Maiguma M, Suzuki Y, Suzuki H, Okazaki K, Aizawa M, Muto M, et al. Dietary zinc is a key environmental modifier in the progression of IgA nephropathy. PLoS One. 2014;9(2): e90558.

3 Fan P, Song J, Chen Q, Cheng X, Liu X, Zou $\mathrm{C}$, et al. The influence of environmental factors on clinical pathological changes of patients with immunoglobulin A nephropathy from different areas of China. Ren Fail. 2018 Nov;40(1):597-602.

4 Elboudwarej E, Cole M, Briggs FB, Fouts A, Fain PR, Quach H, et al. Hypomethylation within gene promoter regions and type 1 diabetes in discordant monozygotic twins. J Autoimmun. 2016 Apr;68:23-9.

5 Nakano K, Whitaker JW, Boyle DL, Wang W, Firestein GS. DNA methylome signature in rheumatoid arthritis. Ann Rheum Dis. 2013 Jan;72(1):110-7.

6 Sun Q, Zhang J, Zhou N, Liu X, Shen Y. DNA methylation in Cosmc promoter region and aberrantly glycosylated IgA 1 associated with pediatric IgA nephropathy. PLoS One. 2015; 10(2): 0112305.

7 Sallustio F, Serino G, Cox SN, Dalla Gassa A, Curci C, De Palma G, et al. Aberrantly methylated DNA regions lead to low activation of CD4+ T-cells in IgA nephropathy. Clin Sci. 2016 May;130(9):733-46.

8 Hayashi K, Hishikawa A, Hashiguchi A, Azegami T, Yoshimoto N, Nakamichi R, et al. Association of glomerular DNA damage and DNA methylation with one-year eGFR decline in IgA nephropathy. Sci Rep. 2020 Jan 14;10(1):237.
9 Xia M, Chen G, Liu D, Tang X, Liu Y, Wu L, et al. Association analysis of DNA methyltransferases in IgA nephropathy. Int Immunopharmacol. 2020 Mar;80:106147.

10 Ahlmen J, Hedman I, Svalander C. Recurrent IgA-nephropathy in an identical twin transplant. Clin Transpl. 1989;297.

11 Tsuboi N, Kawamura T, Okonogi H, Ishii T, Utsunomiya Y, Hosoya T. Discordant clinicopathological features in monozygotic twins with IgA nephropathy. Nephrol Dial Transplant. 2011 Dec;26(12):4146-8.

12 Irvine J, Cross N, Lynn K, Robson R, Searle M, Morton J, et al. IgA nephropathy in identical twins: an excellent donor and recipient outcome 11 years after transplantation. Clin Kidney J. 2012 Jun;5(3):232-3.

13 Miller SA, Dykes DD, Polesky HF. A simple salting out procedure for extracting DNA from human nucleated cells. Nucleic Acids Res. 1988 Feb 11;16(3):1215.

14 Aryee MJ, Jaffe AE, Corrada-Bravo H, LaddAcosta C, Feinberg AP, Hansen KD, et al. Minfi: a flexible and comprehensive Bioconductor package for the analysis of Infinium DNA methylation microarrays. Bioinformatics. 2014 May 15;30(10):1363-9.

15 Pidsley R, CC YW, Y Wong CC, Lunnon K, Mill J, Schalkwyk LC. A data-driven approach to preprocessing Illumina $450 \mathrm{~K}$ methylation array data. BMC Genomics. 2013 May 1;14: 293.

16 Peters TJ, Buckley MJ, Statham AL, Pidsley R, Samaras K, Lord RV, et al. De novo identification of differentially methylated regions in the human genome. Epigenetics Chromatin. 2015;8:6.
17 V Lord MA, van der Zwan YG, Dorssers LC, Looijenga LH. DMRforPairs: identifying differentially methylated regions between unique samples using array based methylation profiles. BMC Bioinformatics. 2014 May 15;15:141.

18 Yu G, Wang LG, He QY. ChIPseeker: an R/ Bioconductor package for ChIP peak annotation, comparison and visualization. Bioinformatics. 2015 Jul 15;31(14):2382-3.

19 Liao Y, Wang J, Jaehnig EJ, Shi Z, Zhang B. WebGestalt 2019: gene set analysis toolkit with revamped UIs and APIs. Nucleic Acids Res. 2019 Jul 2;47(W1):W199-w205.

20 Park JS, Jeong JH, Byun JK, Lim MA, Kim EK, Kim SM, et al. Regulator of calcineurin 3 ameliorates autoimmune arthritis by suppressing Th17 cell differentiation. Am J Pathol. 2017 Sep;187(9):2034-45.

21 Peters VA, Joesting JJ, Freund GG. IL-1 receptor 2 (IL-1R2) and its role in immune regulation. Brain Behav Immun. 2013 Aug;32: $1-8$

22 Xie M, Zhang D, Zhang Y, Yang X, Su Y, Wang Y, et al. Association of genetic polymorphisms in IL-1R1 and IL-1R2 genes with IgA nephropathy in the Han Chinese population. Oncotarget. 2017 Aug 1;8(31):50673-9.

23 Satoh T, Akira S. Toll-like receptor signaling and its inducible proteins. Microbiol Spectr. 2016 Dec;4(6).

24 Coppo R, Camilla R, Amore A, Peruzzi L, Daprà V, Loiacono E, et al. Toll-like receptor 4 expression is increased in circulating mononuclear cells of patients with immunoglobulin A nephropathy. Clin Exp Immunol. 2010 Jan;159(1):73-81.

25 Suzuki H, Suzuki Y, Narita I, Aizawa M, Kihara M, Yamanaka T, et al. Toll-like receptor 9 affects severity of IgA nephropathy. J Am Soc Nephrol. 2008 Dec;19(12):2384-95. 
26 Ciferska H, Honsova E, Lodererova A, Hruskova Z, Neprasova M, Vachek J, et al. Does the renal expression of toll-like receptors play a role in patients with IgA nephropathy? J Nephrol. 2020 Apr;33(2):307-16.

27 Pierik M, Joossens S, Van Steen K, Van Schuerbeek N, Vlietinck R, Rutgeerts P, et al. Toll-like receptor-1, -2, and -6 polymorphisms influence disease extension in inflammatory bowel diseases. Inflamm Bowel Dis. 2006 Jan;12(1):1-8.

28 Kuai J, Gregory B, Hill A, Pittman DD, Feldman JL, Brown T, et al. TREM-1 expression is increased in the synovium of rheumatoid arthritis patients and induces the expression of pro-inflammatory cytokines. Rheumatology. 2009 Nov;48(11):1352-8.
29 Schenk M, Bouchon A, Seibold F, Mueller C. TREM-1: expressing intestinal macrophages crucially amplify chronic inflammation in experimental colitis and inflammatory bowel diseases. J Clin Invest. 2007 Oct;117(10): 3097-106.

30 Ford JW, McVicar DW. TREM and TREMlike receptors in inflammation and disease. Curr Opin Immunol. 2009 Feb;21(1):38-46.

31 Zhu L, Zhang Q, Shi S, Liu L, Lv J, Zhang H. Synergistic effect of mesangial cell-induced CXCL1 and TGF- $\beta 1$ in promoting podocyte loss in IgA nephropathy. PLoS One. 2013; 8(8):e73425.

32 Zhao YF, Zhu L, Liu LJ, Shi SF, Lv JC, Zhang H. TREM-1 contributes to inflammation in IgA nephropathy. Kidney Dis. 2018 Feb;4(1): 29-36.

33 Sao T, Yoshino Y, Yamazaki K, Ozaki Y, Mori $\mathrm{Y}$, Ochi S, et al. TREM1 mRNA expression in leukocytes and cognitive function in Japanese patients with Alzheimer's disease. J Alzheimers Dis. 2018;64(4):1275-84.
34 Ruszkowski J, Lisowska KA, Pindel M, Heleniak Z, Dębska-Ślizień A, Witkowski JM. T cells in IgA nephropathy: role in pathogenesis, clinical significance and potential therapeutic target. Clin Exp Nephrol. 2019 Mar; 23(3):291-303

35 Lin FJ, Jiang GR, Shan JP, Zhu C, Zou J, Wu $\mathrm{XR}$. Imbalance of regulatory $\mathrm{T}$ cells to Th17 cells in IgA nephropathy. Scand J Clin Lab Invest. 2012 May;72(3):221-9.

36 Peng Z, Tian J, Cui X, Xian W, Sun H, Li E, et al. Increased number of Th22 cells and correlation with Th17 cells in peripheral blood of patients with IgA nephropathy. Hum Immunol. 2013 Dec;74(12):1586-91.

37 Yang L, Zhang X, Peng W, Wei M, Qin W. MicroRNA-155-induced T lymphocyte subgroup drifting in IgA nephropathy. Int Urol Nephrol. 2017 Feb;49(2):353-61. 